Archives de sciences sociales des religions

148 | octobre-décembre 2009

Bulletin Bibliographique

\title{
À propos de la notion d'expulsion
}

\section{Danièle Dehouve}

\section{(2) OpenEdition}

Journals

Édition électronique

URL : http://journals.openedition.org/assr/21471

DOI : 10.4000/assr. 21471

ISSN : $1777-5825$

Éditeur

Éditions de l'EHESS

\section{Édition imprimée}

Date de publication : 31 décembre 2009

Pagination : 25-31

ISBN : 978-2-7132-2218-4

ISSN : 0335-5985

\section{Référence électronique}

Danièle Dehouve, «À propos de la notion d'expulsion », Archives de sciences sociales des religions [En ligne], 148 | octobre-décembre 2009, mis en ligne le 01 janvier 2013, consulté le 30 avril 2019. URL http://journals.openedition.org/assr/21471; DOI : 10.4000/assr.21471 


\section{Danièle Dehouve}

\section{À propos de la notion d'expulsion}

Avec la réconciliation du sacré et du symbolique, l'ouvrage de Camille Tarot ouvre, à mon sens, un espace théorique fécond et jette les bases d'un passionnant projet de recherche en anthropologie comparative. Il devrait permettre de poser (ou reposer) des questions qui ne trouvaient pas leur place dans l'approche structuraliste du «tout symbolique ». Parmi celles-ci, les actes rituels d'expulsion (dont l'objectif est l'élimination d'un élément jugé négatif par l'individu ou le groupe social) posent des problèmes fondamentaux. La notion apparaît tout d'abord sous la plume d'Hubert et Mauss sous l'appellation de sacrifice d'expiation ou de désacralisation. Elle disparaît ensuite de l'anthropologie française, à l'exception des travaux de René Girard qui en fait le socle de sa théorie de la victime émissaire. En quelque sorte, l'expulsion se trouve au cœur du conflit entre Girard et Lévi-Strauss que Tarot a nommé la "grande guerre ». Ceci n'est pas pour faciliter la reprise du dossier : il nous faut repartir de l'héritage de Mauss, prendre position par rapport à la théorie girardienne et, surtout, repérer les pratiques rituelles d'expulsion dans les données de terrain. C'est dans les sociétés indiennes du Mexique ancien (chez les Aztèques) et moderne (dans les communautés indiennes contemporaines) que j'ai commencé à aborder la question de l'exclusion rituelle il y a quelques années (Dehouve, 2006) et, depuis, je n'ai cessé d'être frappée par son omniprésence.

\section{La double articulation du sacré}

Toute discussion sur la notion d'expulsion doit partir du cadre offert par la définition maussienne du sacré, comme catégorie pratique du rituel, appartenant au registre du performatif, et marquée par la double articulation sacré/profane et pur/impur. "Le sacrifice est l'acte religieux qui, par la consécration d'une victime, modifie l'état de la personne morale qui l'accomplit ou de certains objets auxquels elle s'intéresse »(Hubert, Mauss, 1968). De là provient la première articulation - l'opposition entre le profane et le sacré - qui détermine le schème du rite, avec des phases d'entrée et de sortie.

La seconde articulation est déduite de la distinction entre deux types de sacrifices, dits « de consécration » lorsque le caractère qui fait l'objet du rite se transmet 
de la victime au sacrifiant, et "d'expiation » lorsque celui-ci va du sacrifiant à la victime. Sous le terme d'expiation, Hubert et Mauss désignent l'élimination pure et simple (p. 258) ou l'expulsion d'un élément dont on cherche à se " débarrasser» (p. 261), qui peut être aussi bien la maladie et la mort que le péché. Si l'on peut juger peu heureux le terme "expiation» qui a déclenché bien des polémiques, il n'en reste pas moins que les auteurs ont posé que la même pratique sacrificielle pouvait être mise au service de deux objectifs apparemment opposés, la consécration et l'exécration, l'obtention et l'élimination.

\section{Le tout symbolique}

La notion de sacré a, par la suite, fait les frais d'une vigoureuse attaque structuraliste. Aujourd'hui encore on peut lire sous la plume d'Alain Caillé : «la catégorie du sacré est elle-même trop incertaine et contestée pour qu'il soit possible d'en faire un usage non problématique. Avant de l'abandonner comme tous les chercheurs contemporains... » (2000: 148). Ces mots sont étonnants pour qui travaille dans un milieu anthropologique international où la plupart des chercheurs utilisent le terme de sacré sans état d'âme particulier. Il faut chercher les raisons de cette perception proprement hexagonale dans un événement franco-français : l'offensive structuraliste des années soixante à soixante-dix.

Tarot (2008 : 385-395) a parfaitement décrit la façon dont Lévi-Strauss a opposé sa théorie symbolique au sacré durkheimien et maussien. Après ses premiers travaux envisageant le totémisme, non comme une religion, mais comme un système classificatoire, Lévi-Strauss réitère la démonstration à propos des mythes. Cependant, les rites se laissent difficilement aborder comme des systèmes de classification et, dans quelques pages célèbres de la Pensée sauvage et de L'Homme nu, Lévi-Strauss voit dans le sacrifice un opérateur anti-classificatoire, un mélangeur de catégories. Lui-même ne développera pas cette question plus avant et ce sont deux autres chercheurs qui tenteront de démontrer que les sacrifices peuvent également être envisagés comme des systèmes classificatoires. Ce sont eux également qui mettront en exergue de leurs travaux la critique de l'Essai d'Hubert et Mauss.

Contentons-nous de mentionner le premier, Marcel Detienne (1979), dont la démonstration porte sur le sacrifice chez les anciens grecs. Le second, Luc de Heusch (1986), se distingue par la sophistication de son analyse du sacrifice africain comme résultat du système classificatoire.

Pour de Heusch, la totalité de la vie sociale se fonde sur un ordre symbolique, séparant des catégories - comme le monde surnaturel et le monde humain, le ciel et la terre, certains parents séparés par le tabou de l'inceste - et interdisant leur contact. Si celui-ci se produit, il entraîne un dérèglement et des maladies. C'est alors qu'intervient le sacrifice, comme moyen de rétablir la distance nécessaire : "l'intervention du religieux vient après : elle est une sanction qui frappe celui qui a enfreint la règle, que l'infraction ait été délibérée ou non» $(1986: 23)$. 
Le religieux est donc considéré comme un simple épiphénomène du système symbolique entendu comme système classificatoire au fondement de la vie sociale.

Si le point de vue de de Heusch nous intéresse particulièrement ici, c'est qu'il le conduit à refuser la notion d'expulsion, largement utilisée avant lui par EvansPritchard (1956) dans son analyse classique de la religion nuer. Dans ce but, de Heusch écarte le fait que les Nuers eux-mêmes possèdent le terme woc (getting rid of something, to move, to put away, take away of anything) qui désigne un acte rituel dont le but est d'évacuer un caractère négatif. À ses yeux, le sacrifice vise seulement à rétablir la séparation entre des mondes malencontreusement placés en situation de contiguité dans la personne du malade.

L'entreprise de de Heusch ne peut être comprise en dehors de son cadre théorique : sur le modèle de l' «illusion totémique », il veut prouver que le sacrifice n'a pas d'existence en dehors d'un système de relations symboliques. Et c'est également la raison de l'explication qu'il donne à la mise à mort rituelle du roi africain. Loin d'être une victime émissaire, dit-il, celui-ci n'est considéré comme " un être dangereux, entouré d'interdits et condamné à une mort prématurée " que parce qu'il confond en sa personne des espaces symboliques qui devraient rester séparés : l'espace humain et l'espace de brousse (cité par Tarot, 2008 : 639).

À l'encontre de ces analyses qui me semblent considérablement biaisées par la volonté de rabattre le religieux sur le symbolique et l'expulsion sur le classificatoire, je pense qu'il y a effectivement des choses dont les groupes sociaux veulent se débarrasser et des actes rituels dont c'est le but.

\section{L'expulsion chez les Indiens mexicains}

Lorsqu'on a commencé à les repérer, les actes rituels d'expulsion pratiqués par les anciens Aztèques ou les Indiens contemporains s'avèrent étonnamment nombreux. Les actuels Indiens tlapanèques du sud-ouest du Mexique donnent le nom de kwei, traduit en espagnol par mal aire ("mauvais air»), à tout un ensemble de phénomènes considérés comme néfastes : la maladie, les mauvaises relations sociales, le vol et la famine. À chacun son propre rituel d'expulsion : on peut confectionner un dépôt rituel surmonté d'animaux sacrifiés ou réaliser une limpia en frottant une personne avec des herbes; la famine est représentée par un mannequin et symboliquement jetée dans le fleuve à la fin de la période de soudure alimentaire. Pour éviter que les animaux nuisibles ne détruisent les champs de maïs, on capture des rats et des oiseaux et on mime une invitation au cours de laquelle on les accueille en hôtes d'honneur, on les enivre et on danse avec eux jusqu'à ce qu'ils périssent écrasés sous les pieds des danseurs.

Dans le Mexique central, ancien et moderne, plusieurs techniques sont utilisées pour chasser la grêle ; on fait éclater des pétards pour lui "faire peur » et des spécialistes ont pour fonction de la détourner vers des villages voisins ou des 
lieux inhabités au moyen de leur souffle. Partout, les morts sont conçus comme porteurs de mauvaises influences qu'il s'agit de contrôler par une grande variété de rituels.

Comme il est impossible de développer ici ce vaste sujet, on dira seulement que les moyens rituels de l'expulsion sont de deux sortes. D'une part, les moyens métaphoriques : on capture l'élément néfaste au moyen du souffle ou du balayage et on l'enferme dans un support - un animal, un mannequin ou des guirlandes de fleurs - que l'on jette dans un endroit désert ou sur le territoire voisin. La principale métaphore de l'expulsion est le déplacement : on ne fait pas disparaitre l'élément négatif, on l'envoie ailleurs. En dernière instance, la source est la grande recycleuse car elle emporte les choses au loin dans son courant. Il faut remarquer que le sacrifice de l'animal entraîne le déplacement des éléments négatifs dans l'au-delà ; la mort de la victime représente donc une façon comme une autre de se débarrasser des influences néfastes qui ont été auparavant capturées dans son corps.

Le second moyen de l'expulsion est le don disjonctif. Il peut ressembler en tout point au don d'obtention : on offre à la puissance considérée (ici une puissance néfaste) des assemblages de fleurs et un sacrifice, à ceci près que la contrepartie demandée est son départ. La métaphore rituelle du déplacement se conjugue très souvent à un don disjonctif, comme dans l'exemple donné plus haut de l'invitation des rats et des oiseaux qui s'achève par leur mort. Dans un sacrifice d'expulsion, l'animal représente à la fois un don à une puissance considérée et un moyen métaphorique de déplacement vers l'au-delà.

Il y a cependant quelque chose de troublant dans les rituels mexicains. C'est leur capacité à exprimer conjointement l'obtention et l'expulsion. Lors d'un rituel observé par l'ethnologue Guy Stresser-Péan $(2005$ : 162) chez les Indiens totonaques du sud-est du Mexique, les poulets absorbent les impuretés des sacrifiants avant d'être offerts aux divinités et consommés par les membres du groupe. Le même acte sacrificiel est donc investi de significations qui peuvent nous paraître contradictoires : l'expulsion d'éléments nocifs et dangereux par transfert sur la victime est suivie de l'utilisation de la même victime dans le cadre d'un rituel d'obtention. En raison de ce que l'on peut nommer la polysémie ou la condensation rituelle, ce type de cérémonies à double fonction est très répandu au Mexique.

Cependant, la notion d'expulsion n'est pas réservée au seul domaine rituel. Elle s'inscrit dans la structure sociale et s'incarne dans le personnage aux fonctions à la fois politiques et rituelles qui se trouve au centre d'un système à royauté sacrée. J'ai montré (Dehouve, 2006) que si les rois sacrés de l'ancien Mexique ont disparu avec la Conquête espagnole, leur figure survit au sein de l'organisation politico-religieuse des actuelles communautés indiennes. Sous des noms variés empruntés à la législation nationale (maire, alcalde...), il existe toujours dans les villages un ou plusieurs personnages dont les fonctions sont ambivalentes. Pour 
une part, ils font régner la justice et président aux cérémonies; mais, pour une autre, ils font pénitence pour le bien-être du village; au moyen du jeûne, de l'abstinence sexuelle et de la veille, ils "demandent pardon » au nom de leurs concitoyens. En quelque sorte, ils absorbent les éléments négatifs produits par le groupe et l'en débarrassent. Dans l'ancien Mexique, ces fonctions pénitentielles s'accompagnaient de pénibles saignées. En cas de malheur - mort des habitants, épidémies -, le responsable tout trouvé en est ce personnage accusé de ne pas avoir accompli correctement ses pénitences.

\section{Le bouc émissaire girardien}

Il est temps de se demander si la théorie girardienne du bouc émissaire est susceptible de rendre compte de ces phénomènes d'expulsion rituelle. Pour René Girard, l'ordre culturel réside dans un système organisé de différences ; leur perte déclenche la crise sacrificielle ou crise d'indifférenciation, qui est également un état de conflit social. La mise à mort du bouc émissaire arrête la violence et rétablit l'ordre au moyen du "processus victimaire ». Le bouc émissaire change alors de nature et, de monstre, se métamorphose en sauveur du groupe. En conséquence, le sacré représente la violence projetée sur le bouc émissaire et expulsée de la communauté, il est avant tout séparation et éloignement.

Il est vrai que cette théorie est restée la seule à s'intéresser aux phénomènes d'expulsion rituelle à une période où ceux-ci étaient niés par les courants dominants. Deux de ses présupposés font cependant problème. Girard assure, d'une part, que l'objet de l'expulsion est la violence interne au groupe et, de l'autre, proclame l'antériorité de l'expulsion sur l'obtention. Les données mexicaines, à l'inverse, montrent qu'il existe de nombreuses choses qu'un groupe social peut vouloir expulser, pas seulement les mauvaises relations sociales; d'autre part, elles mettent au défi de concevoir la simultanéité des opérations d'obtention et d'expulsion. Le système rituel et social repose sur la conception d'une ambivalence généralisée. Chaque phénomène naturel a deux faces : la pluie est bénéfique mais la grêle, destructrice ; la croissance du maïs est désirable mais elle attire les animaux nuisibles. Tous les phénomènes sociaux témoignent de la même ambivalence : le responsable politique qui rend la justice participe à l'ordre de l'univers, mais il provoque en même temps le ressentiment de ses concitoyens. Bref, pour répondre à cette ambivalence généralisée, il convient de pratiquer conjointement des rituels d'obtention et d'expulsion. De même, la double face du responsable politique, comme souverain en majesté et victime pénitentielle, provient du fait qu'il concentre en sa personne la prospérité et l'adversité.

Dans mon étude des prières des Indiens tlapanèques, j'ai constaté que la moitié des souhaits exprimés concerne ce que l'on veut (la pluie, la fertilité, la croissance, la tranquillité...) et l'autre moitié, ce que l'on ne veut pas (la grêle, la maladie, la mort, les bagarres...). On peut en déduire que les demandes 
s'expriment sur le mode positif et négatif. L'acte rituel est intrinsèquement polysémique.

Le jeu de l'obtention et de l'expulsion, in fine, porte sur la vie et la mort. Hocart a donné de la "vie » une large définition incluant la santé, la fertilité et la prospérité. On peut, de la même façon, définir la « mort » comme un ensemble de phénomènes néfastes comprenant la famine, la maladie, la transgression et les mauvaises relations sociales. Les rituels sont conçus comme des façons d'obtenir la vie (c'est leur composante positive, l'obtention) et de rejeter la mort - et souvent les morts eux-mêmes - (c'est leur composante négative, l'expulsion).

La conception girardienne appauvrit la notion d'expulsion en la restreignant à la structure du "tous contre un ". L'expulsion permet d'analyser des phénomènes autrement complexes que le lynchage du bouc émissaire. Tarot (2008 : 810-816) commente longuement la description d'un village à castes du nord de l'Inde (Raheja, 1988). Or Raheja place au cœur de son analyse les catégories faste/néfaste. La caste centrale du village pratique quotidiennement des dons aux brahmanes, mais ce sont des dons empoisonnés par le néfaste qui portent le nom de dan ; les brahmanes, eux, sont obligés de les accepter et, ce faisant, prennent sur eux les afflictions et les fautes du donneur, puis mettent en pratique certains moyens visant à assimiler et décomposer ces substances. Cette importante contribution met en évidence des moyens d'expulsion que la théorie du bouc émissaire n'a pas prévus : une catégorie spéciale de don disjonctif, le don empoisonné, jointe à la pratique de l'ascétisme conçu comme une sorte de système social de recyclage du néfaste.

L'ouvrage de Tarot présente le double intérêt de restituer le contexte théorique des débats qui ont traversé l'anthropologie française et de rendre sa place au sacré. Il faut aujourd'hui dépasser les blocages idéologiques liés à la polémique entre Lévi-Strauss et Girard, éliminer les tabous qu'elle a suscités et repartir de l'observation anthropologique. Dans ce cadre, j'ai essayé de lancer des pistes sur l'expulsion, notion opératoire qui met en lumière des pratiques rituelles et sociales fondamentales. Elle exige cependant d'être plus amplement travaillée et réfléchie. Car, si elle présente l'avantage de dépasser la vision réductrice liée au syndrome émissaire, elle appartient à une catégorie d'analyse encore plus large - la disjonction ou séparation - qui appelle de plus amples réflexions.

Danièle DEHOUVE

Université Paris Ouest La Défense Nanterre / l'EPHE - Sorbonne daniele.dehouve@g.mail.com 


\section{Bibliographie}

CAILlé Alain, 2000, Anthropologie du don. Le tiers paradigme, Paris, Desclée de Brouwer. De Heusch Luc, 1986, Le sacrifice dans les religions africaines, Paris, Gallimard.

Dehouve Danièle, 2006, Essai sur la royanté sacrée en République mexicaine, Paris, CNRS Éditions.

-, 2007, Offrandes et sacrifice en Mésoamérique, Paris, Riveneuve Éditions.

Dehouve Danièle, Prost Richard, 2004, Les dangers du pouvoir, film de 54 mn, Tonaltepec Production tonaltepec@yahoo.fr.

Dehouve Danièle, Vié-Wohrer Anne-Marie, 2008, Le monde des Aztèques, Paris, Riveneuve Éditions.

Detienne Marcel, Vernant Jean-Pierre, 1979, La cuisine du sacrifice en pays grec, Paris, Gallimard.

Evans-Pritchard Edward E., 1956, Nuer Religion, Oxford, The Clarendon Press.

GIRARD René, 1972, La violence et le sacré, Paris, Grasset.

HuberT Henri, Mauss Marcel, 1968, «Essai sur la nature et la fonction du sacrifice ", in Mauss M., Euvres, I, pp. 193-307, Paris, Éditions de Minuit.

Raheja Gloria Goodwin, 1988, The Poison of the Gift. Ritual, Prestation and Dominant Caste in a North Indian Village, Chicago - London, The University of Chicago Press.

Stresser-Pean Guy, 2005, Le Soleil-Dieu et le Christ. La christianisation des Indiens du Mexique, Paris, L'Harmattan.

TARot Camille, 2008, Le symbolique et le sacré. Théories de la religion, Paris, MAUSS/ La Découverte. 
